COMMUNICATIONS IN

ANALYSIS AND GEOMETRY

Volume 7, Number 3, 583-608, 1999

\title{
Geometric Residue Theorems for Bundle Maps
}

\author{
SUNIL NAIR
}

In this paper we prove geometric residue theorems for bundle maps over a compact manifold. The theory developed associates residues to the singularity submanifolds of the map for any invariant polynomial. The theory is then applied to a variety of settings: smooth maps between equidimensional manifolds, CR-singularities, finite singularities and singularities of odd forms as spinor bundle maps.

\section{Introduction.}

The prototype of residue theorems in geometry is the classical theorem of Hopf's $[\mathrm{H}]$ which relates the zeroes of a vector field on a compact manifold to its Euler characteristic. In general, residue theorems associate topological or curvature invariants to the singularities of geometric objects. The aim of this paper is to study singularities of maps between bundles over an oriented manifold, in particular, to obtain residue theorems for such singularities.

The theory of generic bundle maps has been studied in great detail by Harvey-Lawson [HL1], [HL2] and by Harvey-Semmes [HS]. This paper uses several key ideas developed in the papers above, namely the notion of pushforward connections and the universal setting for residue theorems, but takes a different point of view. While the authors above study atomic maps between bundles, for which the singularities are of the expected codimension, we allow the singularities to be nongeneric, asking only that they be closed submanifolds.

A natural class of examples of such bundle maps is the following: Let $X$ be a compact riemannian manifold equipped with an action of a finite group $\Gamma$. Suppose that for some $g \in \Gamma, X / \mathbb{Z}^{m}$ is a smooth manifold, where $\mathbb{Z}^{m}$ is the cyclic subgroup generated by $g$. Let $p: X \mapsto X / \mathbb{Z}^{m}$ be projection. Then the differential map $d p: T X \mapsto p^{*} T X / \mathbb{Z}^{m}$ is a bundle map over $X$ with singularities $F_{g}$, the fixed point set of $g$. By an elementary exponential map argument one can see that $F_{g}$ is a union of smooth, closed submanifolds of $X$, possibly of different dimensions (see [S] for a large collection of examples). This class includes branched coverings over a smooth codimension 2 branch locus. 
The main result of the paper can be summarized as follows. Let $\alpha: E \mapsto$ $F$ be a vector bundle map between vector bundles $E$ and $F$ with connection over a compact, oriented manifold $X$. Suppose that $\alpha$ is injective on $X-\Sigma$, where $\Sigma$ is a union of smooth closed submanifolds $\Sigma_{i}$, possibly of different dimensions. Let $I=\operatorname{Im}(\alpha)$ be the image subbundle of $\alpha$ in $F$. Suppose also that $\alpha$ is normalizable at each $\Sigma_{i}$, in a sense to be described later. Then for each invariant polynomial $\phi$, there is an equation between currents on $X$ :

$$
\phi\left(\Omega_{F}\right)-\phi\left(\Omega_{E} \oplus \Omega_{I^{\perp}}\right)=\sum \operatorname{Res}_{\phi, i}\left[\Sigma_{i}\right]+d T .
$$

Here $\phi(\Omega)$ denotes the $\phi$-characteristic form of a connection, $T$ is an $L_{l o c}^{1}$ transgression form on $X$ which is smooth on $X-\Sigma$ and the residue $R_{e s} s_{\phi, i}$ is a smooth form on $\Sigma$ defined by the fiber integral

$$
\operatorname{Res}_{\phi, i}=-\int_{\left.\pi_{i}\right|_{\partial N_{i, \epsilon}}} T
$$

where $\partial N_{i, \epsilon}$ is the boundary of an $\epsilon$-tubular neighborhood $\pi: N_{i, \epsilon} \mapsto \Sigma_{i}$ of $\Sigma_{i}$. Equation (1.1) should be viewed as a natural generalization of the Poincaré-Hopf Index Theorem to vector bundle maps.

The concept of normalization can be briefly described as follows. A vector bundle map $\alpha: E \mapsto F$ is called normalizable at $\Sigma$ if $\alpha$ and the connections of $E$ and $F$ are radially constant with respect to the natural radial projection $\rho: N_{\epsilon}-\Sigma \mapsto \partial N_{\epsilon}$. Normalization is not a strong condition because we show that any bundle map which is injective outside a union of closed, smooth submanifolds $\Sigma$ is homotopic to a map which is normalized at $\Sigma$. Furthermore, at the cohomology level, equation (1.1) is homotopy invariant when $\operatorname{rank}(\mathrm{E})=\operatorname{rank}(\mathrm{F})$.

This approach leads to many interesting formulae and applications. To name a few, we obtain a generalized Hopf index theorem for bundle maps with finite singularities, a generalized Riemann-Hurwitz formula for smooth maps between manifolds of the same dimension, residue formulae for CRsingularities and residue formulae for Clifford and spin bundles.

The author wishes to thank Blaine Lawson for introducing him to the subject and for his invaluable help in shaping the results obtained in his thesis, from which this paper stems. Also, he wishes to thank ICTP, where a part of this work was realized.

\section{Pushforward Connections.}

In this section we review the concept of a pushforward connection introduced in [HL1]. The material covered in $\S 2$ to $\S 4$ can be found in the above 
paper in much greater detail.

A notational convention: Throughout this paper $X$ will denote a manifold which is oriented and $\Sigma$ a submanifold of $X$.

Suppose $E$ and $F$ are vector bundles (real or complex) over an oriented manifold $X$. Let $D_{E}$, respectively $D_{F}$, be a smooth connection on $E$, respectively $F$. Let $\alpha$ be a bundle map between $E$ and $F$ that is injective outside a submanifold $\Sigma$ of $X$. Then we can transplant the connection $D_{E}$ to define a pushforward connection on $F$ outside the singularity set $\Sigma$ by

$$
\vec{D}=\alpha D_{E} \beta+D_{F}(1-\alpha \beta) .
$$

Here $\beta$ is the 'inverse of $\alpha$ '. This is made precise below. Suppose $E$ and $F$ are equipped with metrics, not necessarily compatible with the connections $D_{E}$ and $D_{F}$. On the complement of $\Sigma$ let $I=\operatorname{Im}(\alpha)$ denote the image subbundle of $F$. We can now choose $\beta$ to be the orthogonal projection of $F$ onto $I$ followed by the inverse of the map

$$
\alpha: E \mapsto I .
$$

The transplanted connection $\vec{D}$ is singular because the map $\beta$ is singular on $\Sigma$. A more concrete formula for $\beta$ is given by

$$
\beta=\left(\alpha^{*} \alpha\right)^{-1} \alpha^{*}
$$

where $\alpha^{*}$ denotes the adjoint of $\alpha$.

For the most part, this paper will concentrate on the equirank case, i.e, when $\operatorname{rank}(\mathrm{E})=\operatorname{rank}(\mathrm{F})$. Then the singular pushforward connection $\vec{D}$ on $F$ is given by the simple formula

$$
\vec{D}=\alpha D_{E} \alpha^{-1} \text { on } X-\Sigma .
$$

On $X-\Sigma$ the pushforward connection $\vec{D}$ can be written in block form with respect to the splitting $F=I \oplus I^{\perp}$. The matrix form of $\vec{D}$ blocks as an upper triangular matrix with diagonal terms $\alpha D_{E} \beta$ and $(1-P) D_{F}(1-P)$. Here $P$ denotes the orthogonal projection of $F$ onto $I$. Therefore for any Ad-invariant polynomial $\phi$, on the Lie algebra $g \ell_{n}(\mathbb{R})$, or $g \ell_{n}(\mathbb{C})$, we have

$$
\phi(\vec{D})=\phi\left(\alpha D_{E} \beta \oplus(1-P) D_{F}(1-P)\right) \quad \text { on } \quad X-\Sigma
$$

where to simplify notation we write

$$
\phi(D) \equiv \phi(\Omega) .
$$


This denotes the invariant polynomial evaluated on the appropriate curvature. This will be the notational covention adopted throughout the paper unless stated otherwise.

When restricted to sections of $I, \alpha D_{E} \beta=\alpha D_{E} \alpha^{-1}$ is gauge equivalent to $D_{E}$ and this implies that

$$
\phi(\vec{D})=\phi\left(D_{E} \oplus D_{I^{\perp}}\right) \quad \text { on } \quad X-\Sigma
$$

where $D_{I^{\perp}}=(1-P) D_{F}(1-P)$ is the connection induced on $I^{\perp} \subset F$ by $D_{F}$.

\section{Families of Pushforward Connections and Transgressions.}

To obtain a transgression formula relating $\phi(\vec{D})$ to $\phi\left(D_{F}\right)$ via ChernWeil theory we want to introduce a family of connections on $F$. There is a nice way of doing this by using the notion of an approximate one. By an approximate one we mean a function $\chi$ with the following properties:

$$
\chi:[0, \infty] \mapsto[0,1]
$$

which is $C^{\infty}$ on $[0, \infty]$ and satisfies

$$
\chi(0)=0, \chi(\infty)=1
$$

and

$$
\chi^{\prime} \geq 0
$$

Given a bundle map $\alpha$ we can define approximations to the inverse of $\alpha$ based on $\chi$ by setting

$$
\beta_{s}=\frac{\alpha^{*}}{s^{2}} \rho\left(\frac{\alpha \alpha^{*}}{s^{2}}\right)
$$

where $\rho(t)=\frac{1}{t} \chi(t)$.

The family of bundle maps $\beta_{s}$ is smooth for $0<s \leq+\infty$ on $X$ with $\beta_{\infty}=0$ on $X$ and $\beta_{0}=\beta$ on $X-\Sigma$. We can now define a family of smooth connections $\vec{D}_{s}$ over the entire manifold $X$ including $\Sigma$ for $0<s \leq+\infty$ by

$$
\vec{D}_{s}=\alpha D_{E} \beta_{s}+D_{F}\left(1-\alpha \beta_{s}\right) .
$$

Note that $\vec{D}_{\infty}=D_{F}$ on $X$ and $\vec{D}_{0}=\vec{D}$ on $X-\Sigma$. 
We then have a family of curvature forms $\Omega_{s}$ corresponding to the family of connections $\vec{D}_{s}$. Using standard Chern-Weil theory (see [BoC]) we can write

$$
\phi\left(D_{\infty}\right)-\phi(\vec{D})=d T \text { on } \quad X-\Sigma
$$

and using (2.2) we can rewrite this as

$$
\phi\left(D_{F}\right)-\phi\left(D_{E} \oplus D_{I^{\perp}}\right)=d T \text { on } X-\Sigma .
$$

Here $\mathrm{T}$ denotes the transgression form for this family of connections. The explicit form of $T$ is given by

$$
T=\int_{0}^{\infty} \phi\left(\dot{D}_{t} ; \Omega_{t}\right) d t
$$

where $\phi\left(\dot{D}_{t} ; \Omega_{t}\right)=\left.\frac{d}{d s} \phi\left(\Omega_{t}+s D_{t}\right)\right|_{s=0}$ is the complete polarization of $\phi$. The formula for $T$ above should be read as written and not with the notational convention $\phi(D) \equiv \phi(\Omega)$ introduced in $\S 2$. The aim of this paper is to extend equation (3.1) across the singularity set $\Sigma$ to the entire manifold $X$, thereby obtaining residue formulae.

Remark 3.1. We can also consider the case where $\alpha: E \rightarrow F$ is a surjective map outside $\Sigma$. Let $K \equiv \operatorname{Ker}(\alpha)$ be the kernel subbundle of the map $\alpha$. The pullback connection $\overleftarrow{D}$ on $E$ is defined by

$$
\overleftarrow{D}=\beta D_{F} \alpha+(1-\beta \alpha) D_{E}
$$

where $\beta$ denotes the inverse of the map $\alpha: K^{\perp} \mapsto F$. Again, by considering families of connections, we obtain, for any invariant polynomial $\phi$,

$$
\phi\left(D_{E}\right)-\phi\left(D_{F} \oplus D_{K}\right)=d T \quad \text { on } \quad X-\Sigma .
$$

For the sake of clarity we omit mentioning the surjective case explicitly in the exposition that follows. The formulae are the same in both cases; the reader just has to replace (3.1) with (3.2) to obtain the result for the surjective case.

\section{The Universal Setting.}

It is often useful to consider the above setting universally. By this we mean transplanting the given data to $\operatorname{Hom}^{\times}(E, F)$, the bundle of injective maps from $E$ to $F$. 
Let $\pi: \operatorname{Hom}^{\times}(E, F) \mapsto X$ be the projection map onto the manifold $X$. Then we can pull back the bundles $E$ and $F$ by $\pi$ to obtain the bundles $\pi^{*} E$ and $\pi^{*} F$ over $\operatorname{Hom}^{\times}(E, F)$. There is a tautological bundle map

$$
\tilde{\alpha}: \pi^{*} E \mapsto \pi^{*} F
$$

which at a point $\alpha \in \operatorname{Hom}^{\times}\left(E_{x}, F_{x}\right)$ above $x \in X$ is simply defined to be $\alpha$. This tautological map is injective everywhere. We can pull the connections on $E$ and $F$ back to $\pi^{*} E$ and $\pi^{*} F$ and apply Chern-Weil theory to this setting as we did in the previous section. We then have the universal formula

$$
\phi\left(D_{\pi^{*} F}\right)-\phi\left(D_{\pi^{*} E} \oplus D_{\tilde{I}^{\perp}}\right)=d \tilde{T} \quad \text { on } \operatorname{Hom}^{\times}(E, F) .
$$

A smooth bundle map $\alpha: E \mapsto F$ which is injective outside $\Sigma \subset X$ defines a cross-section of $H_{o m} \times(E, F)$ on $X-\Sigma$ and we have that

$$
\alpha^{*}\left(\pi^{*} E\right)=E
$$

and

$$
\alpha^{*}\left(\pi^{*} F\right)=F
$$

over $X-\Sigma$ as bundles with connections. Furthermore,

$$
\alpha^{*}(\tilde{\alpha})=\alpha \quad \text { on } \quad X-\Sigma .
$$

Thus, every case is a pullback of the universal one. In particular, (4.1) pulls back to give (3.1) on $X-\Sigma$.

\section{Residue Formulae.}

In this section we study equation (3.1) in more detail. We show how to obtain residue formulae when both the transgression form $T$ and the characteristic form $\phi\left(D_{F}\right)-\phi\left(D_{E} \oplus D_{I^{\perp}}\right)$ in the equation above extend as $L_{l o c}^{1}$ forms across the singularities of the bundle map $\alpha$.

Suppose that we are in the setting outlined in $\S 3$, where $\alpha: E \mapsto F$ is a bundle map, defined and injective outside $\bigcup \Sigma_{i}$. Each $\Sigma_{i}$ is assumed to be a submanifold of $X$, disjoint from the others, but not necessarily of the same dimension. The submanifolds $\Sigma_{i}$ will be referred to as the singularities of the map $\alpha$. By equation (3.1) we can write down the following:

$$
\phi\left(D_{F}\right)-\phi\left(D_{E} \oplus D_{I^{\perp}}\right)=d T \quad \text { on } \quad X-\bigcup \Sigma_{i} .
$$

Suppose that the transgression form $T$ and the characteristic form $\phi\left(D_{F}\right)-$ $\phi\left(D_{E} \oplus D_{I^{\perp}}\right)$ in the equation above extend as $L_{l o c}^{1}$ forms across the singularities. 
Theorem 5.1. Let $\alpha: E \mapsto F$ be a map which is injective outside $\bigcup \Sigma_{i}$, where each $\Sigma_{i}$ is a closed submanifold of a compact, oriented manifold $X$. Suppose that $T$ and $\phi\left(D_{F}\right)-\phi\left(D_{E} \oplus D_{I^{\perp}}\right)$ extend as $L_{\text {loc }}^{1}$ forms on $X$ for a given invariant polynomial $\phi$. Furthermore, assume that the extension of $\phi\left(D_{F}\right)-\phi\left(D_{E} \oplus D_{I^{\perp}}\right)$ is d-closed on $X$. Then

$$
\phi\left(D_{F}\right)-\phi\left(D_{E} \oplus D_{I^{\perp}}\right)=\sum_{i=1}^{l} \operatorname{Res}_{\phi, i}\left[\Sigma_{i}\right]+d T \quad \text { on } \quad X
$$

where

$$
R e s_{\phi, i} \equiv-\lim _{\epsilon \rightarrow 0} \int_{\left.\pi_{i}\right|_{\partial N_{i, \epsilon}}} T
$$

is a closed current supported on $\Sigma_{i}$ given by fiber integration and

$$
\operatorname{deg}\left(\operatorname{Res}_{\phi, i}\right)=2 \operatorname{deg}(\phi)-\operatorname{codim}\left(\Sigma_{i}\right) .
$$

Here $\partial N_{i, \epsilon}$ denotes the boundary of an $\epsilon$-tubular neighborhood $N_{i, \epsilon}$ of $\Sigma_{i}$ and $\pi_{i}: \partial N_{i, \epsilon} \rightarrow \Sigma_{i}$ is projection.

Proof. Choose $\epsilon$-tubular neigborhoods $N_{i, \epsilon}$ of $\Sigma_{i}$. Write

$$
X=\left(X-\bigcup N_{i, \epsilon}\right) \cup \bigcup N_{i, \epsilon} .
$$

Since $\phi\left(D_{F}\right)-\phi\left(D_{E} \oplus D_{I^{\perp}}\right)$ extends as an $L_{\text {loc }}^{1}$ form on $X$, we have

$$
\begin{aligned}
\lim _{\epsilon \rightarrow 0}\left(\phi\left(D_{F}\right)-\phi\left(D_{E} \oplus D_{I^{\perp}}\right)\right) \wedge[X-\bigcup & \left.N_{i, \epsilon}\right] \\
& =\left(\phi\left(D_{F}\right)-\phi\left(D_{E} \oplus D_{I^{\perp}}\right)\right) \wedge[X] .
\end{aligned}
$$

Then we can write

$$
\begin{aligned}
\left(\phi\left(D_{F}\right)-\phi\left(D_{E} \oplus D_{I^{\perp}}\right)\right) \wedge[X]= & \lim _{\epsilon \rightarrow 0} d T \wedge\left[X-\bigcup N_{i, \epsilon}\right] \\
= & \lim _{\epsilon \rightarrow 0} d\left(T \wedge\left[X-\bigcup N_{i, \epsilon}\right]\right) \\
& -\lim _{\epsilon \rightarrow 0} \sum_{i=1}^{l} T \wedge\left[\partial N_{i, \epsilon}\right] .
\end{aligned}
$$

Here $[X],\left[X-\bigcup N_{i, \epsilon}\right]$ and $\left[\partial N_{i, \epsilon}\right]$ denote the currents associated with $X$, $X-\bigcup N_{i, \epsilon}$ and $\partial N_{i, \epsilon}$ respectively. 
Since $T$ extends as an $L_{l o c}^{1}$ form on $X$, the family $T \wedge\left[X-\bigcup N_{i, \epsilon}\right]$ converges to $T$ extended by zero, as currents on $X$. We now use the following convergence property which can be easily deduced from the characterization of flat cochains found in Federer [F;4.1.19].

If $a_{\epsilon} \rightarrow a$ and $b_{\epsilon} \rightarrow b$ as $L_{l o c}^{1}$ forms, then $d a_{\epsilon}+b_{\epsilon} \rightarrow d a+b$ in flat norm.

Applied here, this implies that $L_{i} \equiv \lim _{\epsilon \rightarrow 0} T \wedge\left[\partial N_{i, \epsilon}\right]$ exists in flat currents on $X$.

Since $\operatorname{supp}\left(L_{i}\right) \subset \Sigma_{i}$, this current is intrinsic to $\Sigma_{i}$, by Federer's Flatness Theorem found in Federer [F;4.1.15], i.e, $\pi_{i *} L_{i}=L_{i}$ where $\pi_{i}: N_{i, \epsilon} \rightarrow \Sigma_{i}$ is the fibration of the tubular neighborhood over $\Sigma_{i}$. Now

$$
\begin{aligned}
L_{i} & =\pi_{i *} L_{i} \\
& =\pi_{i *}\left[\lim _{\epsilon \rightarrow 0} T \wedge \partial N_{i, \epsilon}\right] \\
& =\lim _{\epsilon \rightarrow 0} \pi_{i *}\left[T \wedge \partial N_{i, \epsilon}\right] \\
& =\lim _{\epsilon \rightarrow 0} \int_{\left.\pi_{i}\right|_{\partial N_{i, \epsilon}}} T \wedge\left[\Sigma_{i}\right] \\
& =-\operatorname{Res}_{\phi, i}\left[\Sigma_{i}\right] .
\end{aligned}
$$

We note that if $\Sigma_{i}$ is nonorientable then integration over the fiber defines a current with twisted coefficients in the orientation bundle of $N_{i, \epsilon}$. Since $X$ is orientable, $\operatorname{Res}_{\phi, i}$ and $\left[\Sigma_{i}\right]$ lie in the same orientation class, hence $\operatorname{Res}_{\phi, i}\left[\Sigma_{i}\right]$ is well-defined as a current on $X$.

If $\phi\left(D_{F}\right)-\phi\left(D_{E} \oplus D_{I^{\perp}}\right)$ extends as a closed $L_{l o c}^{1}$ form and each $\Sigma_{i}$ is a closed submanifold then applying the exterior derivative on both sides of (5.1) gives that each $R e s_{\phi, i}$ is a closed current.

Remark 5.2. We now explain integration over the fibers a little more carefully. If $\Sigma_{i}$ is orientable, integration over the fibers gives a closed current $\operatorname{Res}_{\phi, i}$ on $X$ supported on $\Sigma_{i}$, which defines an element of $H^{*}(X ; \mathbb{R})$. Suppose now that $\Sigma_{i}$ is nonorientable. We write $N \Sigma_{i} \oplus T \Sigma_{i}=\left.T X\right|_{\Sigma_{i}}$, where $N \Sigma_{i}$ is the normal bundle to $\Sigma_{i}$. Note that the residue $\operatorname{Res}_{\phi, i}$ is given by integration over the fibers of the $o\left(N \Sigma_{i}\right)$-twisted $\epsilon$-sphere bundle in $N \Sigma_{i}$, where $o\left(N \Sigma_{i}\right)$ denotes the orientation bundle of $N \Sigma_{i}$. Since $X$ is orientable,

$$
o\left(N \Sigma_{i}\right) \otimes o\left(T \Sigma_{i}\right)=\underline{\mathbb{R}}
$$

where $o\left(N \Sigma_{i}\right)$ and $o\left(T \Sigma_{i}\right)$ are the orientation bundles of $N \Sigma_{i}$ and $\Sigma_{i}$ respectively, and $\underline{\mathbb{R}}$ is the trivial bundle. Hence $\Sigma_{i}$ is in the same orientation class 
as $\operatorname{Res}_{\phi, i}$, so the pairing $\operatorname{Res}_{\phi, i}\left[\Sigma_{i}\right]$ defines a closed current on $\Sigma_{i}$. Here $\left[\Sigma_{i}\right]$ denotes the current associated with $\Sigma_{i}$ with twisted coefficients in $o\left(\Sigma_{i}\right)$.

With this in hand, it is clear that when we say currents and differential forms, it is to be understood that we mean currents and differential forms with twisted coefficients when $\Sigma_{i}$ is nonorientable.

The hypotheses of the theorem recur again throughout the paper, so for convenience we make the following definition.

Definition 5.3. We say that a bundle map $\alpha$ with singularities $\Sigma_{i}$ which are closed submanifolds is extendable if for any invariant polynomial $\phi$ the smooth forms $T$ and $\phi\left(D_{F}\right)-\phi\left(D_{E} \oplus D_{I^{\perp}}\right)$ in $X-\bigcup \Sigma_{i}$ extend as $L_{l o c}^{1}$ forms on the manifold $X$.

Remark 5.4. If $\operatorname{rank}(\mathrm{E})=\operatorname{rank}(\mathrm{F})$, then $I^{\perp}$ does not appear in the equation above. Since $D_{E}$ and $D_{F}$ are smooth connections, $\phi\left(D_{F}\right)-\phi\left(D_{E}\right)$ extends as a closed smooth form on $X$. Therefore, the only hypothesis needed in this case is that $T$ extend to be $L_{l o c}^{1}$ on $X$.

\section{Normalized Maps and Normalized Bundles.}

In this section we discuss when the transgression form $T$ and the characteristic form $\phi\left(D_{F}\right)-\phi\left(D_{E} \oplus D_{I^{\perp}}\right)$ extend as $L_{l o c}^{1}$ forms on $X$. This involves the notions of normalized bundles and normalized maps which we define below.

First we define a tubular neighborhood structure of a closed submanifold $\Sigma$ to be an $\epsilon$-tubular neighborhood $N_{\epsilon}$ of $\Sigma$ with a given smooth identification with the normal disk bundle to $\Sigma$. By an abuse of notation we will also use $N$ to denote the normal disk bundle to $\Sigma$. Let $\pi: N \rightarrow \Sigma$ denote the bundle projection and $\rho: N-\Sigma \rightarrow \partial N$ denote the radial projection onto the boundary induced from the vector bundle structure of $\pi: N \rightarrow \Sigma$.

Definition 6.1. We say that a bundle $E$ with connection $D_{E}$ over a manifold $X$ is normalized at a submanifold $\Sigma$, if for some tubular neighborhood structure $N$ of $\Sigma$, the pair $\left(E, D_{E}\right)$ can be written as a pullback from $\Sigma$, i.e.,

$$
\left.\left.\left(E, D_{E}\right)\right|_{N} \equiv \pi^{*}\left(E, D_{E}\right)\right|_{\Sigma}
$$

where $\pi: N \rightarrow \Sigma$ is projection. 
Since $\Sigma$ is the retract of $N$, any bundle over $N$ is equivalent to a pullback of a bundle on $\Sigma$. However, the pullback connection is only homotopic to the original one. The condition that a bundle be normalized at $\Sigma$ ensures that the pullback connection is equal to the original one. If $\Sigma$ is a point on $X$ then the normalization condition implies that $E$ is flat in a neighborhood of the point. In general, normalization can be viewed as 'flatness' in radial directions.

We now go on to define normalized maps.

Definition 6.2. A bundle map $\alpha: E \rightarrow F$ defined over $X-\Sigma$ is normalized at $\Sigma$, a submanifold of $X$, if there exists a tubular neighborhood structure $N$ for which $\left(E, D_{E}\right)$ and $\left(F, D_{F}\right)$ are normalized and such that $\alpha$ is radially constant, i.e.,

$$
\rho^{*}\left(\alpha:\left.\left.E\right|_{\partial N} \rightarrow F\right|_{\partial N}\right)=(\alpha: E \rightarrow F) \text { in } N-\Sigma
$$

where $\rho: N-\Sigma \rightarrow \partial N$ is radial projection onto the boundary.

With these definitions in hand we prove the main extension theorem.

Theorem 6.3. Let $\alpha: E \rightarrow F$ be an injective map outside $\bigcup \Sigma_{i}$. Suppose that $\alpha$ is normalized at each $\Sigma_{i}$. Then for any invariant polynomial $\phi$, the forms $T$ and $\phi\left(D_{F}\right)-\phi\left(D_{E} \oplus D_{I^{\perp}}\right)$ extend as $L_{\text {loc }}^{1}$ forms over the manifold $X$, i.e., $\alpha$ is extendable.

Proof. Since $\alpha$ is normalized at each $\Sigma_{i}$, the family of pushforward connections $\vec{D}_{s}$ is also a pullback from $\partial N_{i, \epsilon}$, i.e.,

$$
\left.\left(F, \vec{D}_{s}\right)\right|_{N_{i, \epsilon}-\Sigma_{i}}=\left.\rho^{*}\left(F, \vec{D}_{s}\right)\right|_{\partial N_{i, \epsilon}} .
$$

Here $N_{i, \epsilon}$ denotes the $\epsilon$-disk bundle of $\Sigma_{i}$ and $\partial N_{i, \epsilon}$ its boundary.

This immediately implies that the transgression form is also a pullback from $\partial N_{i, \epsilon}$, i.e.,

$$
\left.T\right|_{N_{i, \epsilon}-\Sigma_{i}}=\rho^{*}\left(\left.T\right|_{\partial N_{i, \epsilon}}\right) .
$$

Now we want to show that this pullback property implies that $T$ extends as an $L_{l o c}^{1}$ form. Since this is a local property we need only construct a local argument. Without loss of generality we can assume that each $\Sigma_{i}$ is of dimension 0 . 
Let $f: \mathbb{R}^{n}-\{0\} \rightarrow S^{n-1}$ be radial projection and $\varphi$ be a form on $S^{n-1}$. We now have to show that $f^{*} \varphi \in L_{l o c}^{1}\left(\mathbb{R}^{n}\right)$. In coordinates $f(x)=\frac{x}{\|x\|}$ where $x=\left(x_{i}, \ldots, x_{n}\right)$. Then

$$
f^{*} d x^{i}=d\left(\frac{x_{i}}{\|x\|}\right)=\frac{d x_{i}}{\|x\|}-\sum \frac{x_{i} x_{j}}{\|x\|^{3}} d x_{j} .
$$

This is a homogeneous form of degree 0 and the coefficients of $f^{*} d x^{i}$ are bounded by $\frac{n}{\|x\|}$. Therefore the coefficients of $f^{*} d x^{I}$, for a multi-index $I$ are bounded by $\left(\frac{n}{\|x\|}\right)^{|I|}$. Hence it is $L_{l o c}^{1}$ if $|I| \leq n-1$. For any form $\varphi$ on $S^{n-1}$ where

$$
\varphi=\left.\sum a_{I} d x^{I}\right|_{S^{n-1}}, \quad|I| \leq n-1,
$$

the coefficients of $f^{*} \varphi$ are bounded by $\sum \sup \left|a_{I}\right| \frac{c}{\|x\|^{n-1}}$, where $c$ is a constant. Here the sup is taken over the sphere. Hence $f^{*} \varphi$ is $L_{l o c}^{1}$.

Applied to the transgression form this implies that $T$ extends as an $L_{l o c}^{1}$ form.

If $2 \operatorname{deg}(\phi)<\operatorname{dim} X$ then the same argument as above implies that $\phi\left(D_{F}\right)-\phi\left(D_{E} \oplus D_{I^{\perp}}\right)$ extends as an $L_{\text {loc }}^{1}$ form. If $2 \operatorname{deg}(\phi)=\operatorname{dim} X$ then $\phi\left(D_{F}\right)-\phi\left(D_{E} \oplus D_{I^{\perp}}\right)=0$ in $N_{i, \epsilon}-\Sigma_{i}$ since it is a form of degree higher than the dimension of $\partial N_{i, \epsilon}$. Hence it extends by zero as an $L_{l o c}^{1}$ form on $X$.

\section{Residues.}

The residue is in general a current supported on the singularities of the bundle map. However, when $\alpha$ is normalized at the singularities the residue is a smooth form.

Lemma 7.1. Let $\alpha: E \rightarrow F$ be an injective map outside $\bigcup \Sigma_{i}$. Suppose that $\alpha$ is normalized at each $\Sigma_{i}$. Then the residue Res $s_{\phi, i}$ is a smooth differential form supported on $\Sigma_{i}$ and is given by

$$
\operatorname{Res}_{\phi, i} \equiv-\int_{\left.\pi_{i}\right|_{\partial N_{i, \epsilon}}} T
$$

where $\pi_{i}: \partial N_{i, \epsilon} \rightarrow \Sigma_{i}$ is projection. Furthermore if either rankE $=$ rankF or $2 \operatorname{deg}(\phi)=\operatorname{dim} X$ then $\operatorname{Res}_{\phi, i}$ is closed. 
Proof. The normalization condition implies that the transgression form $T$ is a pullback from $\partial N_{i, \epsilon}$. This radial invariance makes $\left.T\right|_{\partial N_{i, \epsilon}}$ essentially independent of $\epsilon$. In particular

$$
\lim _{\epsilon \rightarrow 0} \int_{\left.\pi_{i}\right|_{\partial N_{i, \epsilon}}} T=\int_{\left.\pi_{i}\right|_{\partial N_{i, \epsilon}}} T
$$

for any sufficiently small $\epsilon$. Since $T$ is a smooth form outside the singularities, integrating over the fibers of the projection map $\pi_{i}$ yields a smooth closed form on $\Sigma_{i}$.

As mentioned in Remark 5.4, if $\operatorname{rank}(\mathrm{E})=\operatorname{rank}(\mathrm{F})$, then $\phi\left(D_{F}\right)-\phi\left(D_{E}\right)$ extends as a closed smooth form on $X$, hence $\operatorname{Res}_{\phi, i}$ is closed. Also, as observed in the proof of Theorem 6.3, the normalization of $\alpha$ implies the $\phi\left(D_{F}\right)-\phi\left(D_{E} \oplus D_{I^{\perp}}\right)$ is a pullback from $\partial N_{i, \epsilon}$. If $2 \operatorname{deg}(\phi)=\operatorname{dim} X$ then $\phi\left(D_{F}\right)-\phi\left(D_{E} \oplus D_{I^{\perp}}\right)=0$ in $N_{i, \epsilon}-\Sigma_{i}$, since it is a form of degree higher than the dimension of $\partial N_{i, \epsilon}$. So $\phi\left(D_{F}\right)-\phi\left(D_{E} \oplus D_{I^{\perp}}\right)$ extends by zero to be a closed current and $\operatorname{Res}_{\phi, i}$ is closed.

\section{Homotopy, Normalized Maps, and Normalized Bundles.}

We showed in $\S 6$ that $\alpha, E$ and $F$ had to be normalized at the singularities $\Sigma_{i}$ for $T$ and $d T$ to extend as $L_{l o c}^{1}$ forms over the manifold $X$. We now show that normalization is not a strong condition. More precisely we prove that any bundle $E$ with connection is smoothly homotopic to a normalized bundle at $\Sigma_{i}$ and that any bundle map $\alpha$ with singularities $\Sigma_{i}$ is smoothly homotopic to a normalized map on $X-\bigcup \Sigma_{i}$. We also show that any two normalized maps with singularities $\Sigma_{i}$ are homotopic through normalized maps.

Lemma 8.1. Any pair $\left(E, D_{E}\right)$ is smoothly homotopic to a normalized bundle at a submanifold $\Sigma$ of $X$.

Proof. Let $N_{\epsilon / 2}$ be a tubular neighborhood of $\Sigma$. Define a map $p: X \rightarrow X$ as follows.

$$
p(v)=\lambda(\|v\|) v
$$

where $\lambda:[0, \infty] \rightarrow[0,1]$ is a smooth function with the properties

$$
\begin{aligned}
& \lambda(s)=0 \quad \text { for } \quad s<\epsilon / 2, \\
& \lambda(s)=1 \quad \text { for } \quad s \geq \epsilon
\end{aligned}
$$


and

$$
\lambda^{\prime}(s) \geq 0
$$

Then $\left.p\right|_{N_{\epsilon / 2}}=\pi: N_{\epsilon / 2} \rightarrow \Sigma$, where $\pi$ is projection onto $\Sigma$. Hence $p^{*}\left(E, D_{E}\right)$ is equivalent to $E$ and is normalized at $\Sigma$.

Now let $p_{t}(v)=(1-t) v+t p(v)$ for $0 \leq t \leq 1$. Define

$$
\left(E_{t}, D_{t}\right) \equiv p_{t}^{*}\left(E, D_{E}\right) \text {. }
$$

Then $E_{t} \cong E$ for $0 \leq t \leq 1$ and $p_{1}^{*}\left(E, D_{E}\right)=p^{*}\left(E, D_{E}\right)$. Hence $p_{t}^{*}$ is the required homotopy.

Lemma 8.2. Any bundle map $\alpha: E \rightarrow F$ with singularities $\Sigma_{i}$ is homotopic to a normalized map on $X-\bigcup \Sigma_{i}$.

Proof. Let $N_{i, \epsilon}$ be an $\epsilon$-tubular neighborhood of $\Sigma_{i}$. We first normalize $E$ and $F$ at each $\Sigma_{i}$. Define a map $\rho: X-\bigcup \Sigma_{i} \rightarrow X-\bigcup \Sigma_{i}$ as follows. For $v \in N_{i, \epsilon}-\Sigma_{i}$, set

$$
\rho(v)=l(\|v\|) v
$$

where $l:(0, \infty] \rightarrow[1, \infty]$ is a smooth function with the properties

$$
\begin{aligned}
& l(t)=\frac{1}{t} \quad \text { for } \quad t \leq \epsilon / 2, \\
& l(t)=1 \quad \text { for } \quad t \geq \epsilon
\end{aligned}
$$

and

$$
l^{\prime}(t) \leq 0
$$

Then $\left.\rho\right|_{N_{i, \epsilon / 2}-\Sigma_{i}}: N_{i, \epsilon / 2}-\Sigma_{i} \rightarrow \partial N_{i, \epsilon / 2}$ is radial projection in the sense of Definition 6.2 , and extends smoothly to be the identity outside $N_{i, \epsilon}$. Hence $\rho^{*} \alpha$ is normalized at $\Sigma_{i}$. Now let $\rho_{t}(v)=(1-t) v+t \rho(v)$ for $0 \leq t \leq 1$. Then $\rho_{t}^{*}(\alpha)$ is the required homotopy between $\alpha$ and $\rho^{*} \alpha$ defined on $X-\bigcup \Sigma_{i}$.

By the same argument as above we have the following lemmas.

Lemma 8.3. Let $\alpha_{1}: E \rightarrow F$ and $\alpha_{2}: E \rightarrow F$ be normalized at $\Sigma_{i}$, with the same tubular neighborhood structure such that

$$
\alpha_{1}=\alpha_{2} \quad \text { on } \quad X-\bigcup N_{i, \epsilon} .
$$

Then there is a homotopy between $\alpha_{1}$ and $\alpha_{2}$ on $X-\bigcup \Sigma_{i}$ through normalized bundle maps. 
Lemma 8.4. Let $\left(E_{1}, D_{E_{1}}\right)$ and $\left(E_{2}, D_{E_{2}}\right)$ be normalized at $\Sigma_{i}$, with the same tubular neighborhood structure such that

$$
\left(E_{1}, D_{E_{1}}\right)=\left(E_{2}, D_{E_{2}}\right) \quad \text { on } \quad X-\bigcup N_{i, \epsilon} .
$$

Then there is a homotopy between $\left(E_{1}, D_{E_{1}}\right)$ and $\left(E_{2}, D_{E_{2}}\right)$ on $X$ through normalized bundles.

\section{Invariance of Residues under Homotopy.}

We discuss what happens to the residue when we homotope $\alpha$ through normalized maps at the singularities $\Sigma_{i}$. First we recall a double transgression formula found in [HL1].

Lemma 9.1. Let $D_{s, t}$ be a 2-parameter family of connections, $0 \leq s \leq \infty$, and $a \leq t \leq b$, with $D_{s, a}=D_{a}$ and $D_{s, b}=D_{b}$ for all $0 \leq s \leq \infty$. Then the two transgressions, $T_{1}$ and $T_{0}$, determined by $D_{1, t}$ an $d D_{0, t}$ satisfy

$$
T_{1}-T_{0}=d R
$$

with

$$
R=\int_{a}^{b} \int_{0}^{\infty} \phi\left(\frac{\partial}{\partial t} w_{s, t}, \frac{\partial}{\partial s} w_{s, t} ; \Omega_{s, t}\right) d s d t
$$

where

$$
\phi(A, B ; C)=\left.\frac{\partial^{2}}{\partial s \partial t} \phi(C+s A+t B)\right|_{s=t=0} .
$$

The lemma above allows us to prove the following invariance of the residue classes for the equirank case.

Theorem 9.2. Let $\alpha_{0}: E \rightarrow F$ and $\alpha_{1}: E \rightarrow F$ be normalized maps at singularities $\Sigma_{i}$. Assume that $\operatorname{rank}(E)=\operatorname{rank}(F)$. Then the residues, $\operatorname{Res}_{\phi, i}^{0}$ and $\operatorname{Res}_{\phi, i}^{1}$, define the same cohomology class on $\Sigma_{i}$.

Proof. Since $E$ and $F$ are of the same rank and the normalization conditions are satisfied we have that

$$
\phi\left(D_{F}\right)-\phi\left(D_{E}\right)=\sum_{i=1}^{l} \operatorname{Res}_{\phi, i}^{0}\left[\Sigma_{i}\right]+d T_{0} \quad \text { for } \quad \alpha_{0}
$$


and

$$
\phi\left(D_{F}\right)-\phi\left(D_{E}\right)=\sum_{i=1}^{l} \operatorname{Res}_{\phi, i}^{1}\left[\Sigma_{i}\right]+d T_{1} \quad \text { for } \quad \alpha_{1}
$$

where

$$
\operatorname{Res}_{\phi, i}^{0} \equiv-\int_{\left.\pi_{i}\right|_{\partial N_{i, \epsilon}}} T_{0}
$$

and

$$
\operatorname{Res}_{\phi, i}^{1} \equiv-\int_{\left.\pi_{i}\right|_{\partial N_{i, \epsilon}}} T_{1} .
$$

Note that we are allowed to drop the limit $\lim _{\epsilon \rightarrow 0}$ in the formulae above for the residues $R e s_{\phi, i}^{0}$ and $\operatorname{Res}_{\phi, i}^{1}$ by Lemma 7.1.

By Lemma 8.3 we can write a smooth homotopy $\alpha_{t}$ between $\alpha_{0}$ and $\alpha_{1}$ through normalized maps, hence $T_{t}$ extends as an $L_{l o c}^{1}$ form on $X$ for all $0 \leq t \leq 1$. The initial and end points for the two parameter family of pushforward connections defined by the homotopy are $D_{F}$ and $D_{E}$. Thus we are in the setting of the double transgression lemma above and we can write

$$
T_{0}-T_{1}=d R \quad \text { on } \quad X-\bigcup \Sigma_{i}
$$

as smooth forms on $X-\bigcup \Sigma_{i}$, and hence on $\partial N_{i, \epsilon}$.

Since $R$ is smooth on $\partial N_{i, \epsilon}$ and $\partial N_{i, \epsilon}$ is compact without boundary, the exterior derivative $d$ commutes with fiber integration. Integrating equation (9.1) then gives

$$
\operatorname{Res}_{\phi, i}^{1}-\operatorname{Res}_{\phi, i}^{0}=\int_{\left.\pi_{i}\right|_{\partial N_{i, \epsilon}}} T_{0}-\int_{\left.\pi_{i}\right|_{\partial N_{i, \epsilon}}} T_{1}=\int_{\left.\pi_{i}\right|_{\partial N_{i, \epsilon}}} d R=d \int_{\left.\pi_{i}\right|_{\partial N_{i, \epsilon}}} R .
$$

Hence they define the same cohomology class.

\section{The Universal Transgression Form.}

In $\S 4$ we wrote down the following transgression formula which is valid on $\operatorname{Hom}^{\times}(E, F)$, the bundle of injective maps from $E$ to $F$.

$$
\phi\left(D_{\pi^{*} F}\right)-\phi\left(D_{\pi^{*} E} \oplus D_{\tilde{I}^{\perp}}\right)=d \tilde{T} \quad \text { on } \quad H_{o m}^{\times}(E, F)
$$


where $\pi: \operatorname{Hom}^{\times}(E, F) \rightarrow X$ is the projection map. If $\alpha: E \rightarrow F$ is an injective map outside $\Sigma_{i}$ then the equation above pulls down by $\alpha$ to give

$$
\phi\left(D_{F}\right)-\phi\left(D_{E} \oplus D_{I^{\perp}}\right)=d T \quad \text { on } \quad X-\bigcup \Sigma_{i} .
$$

In particular, the transgression form $T$ is just a pullback of the universal transgression form $\tilde{T}$ outside the singularities, i.e.,

$$
T=\alpha^{*} \tilde{T} \quad \text { on } \quad X-\bigcup \Sigma_{i} .
$$

This implies that the residues can be expressed universally in terms of $\tilde{T}$ and the map $\alpha: X-\bigcup \Sigma_{i} \rightarrow H_{o m} \times(E, F)$, considered as a section of $H_{o m} \times(E, F)$ outside the singularities. More precisely,

$$
\operatorname{Res}_{\phi, i}=-\lim _{\epsilon \rightarrow 0} \int_{\left.\pi_{i}\right|_{\partial N_{i, \epsilon}}} \alpha^{*} \tilde{T} .
$$

In a sense, this is a generalization of the notion of the index of a vector field, in that the residue measures the twisting of the map $\alpha$.

\section{Obstructions.}

The most interesting applications of the main residue formula (5.1) arise when $\operatorname{rank}(\mathrm{E})=\operatorname{rank}(\mathrm{F})$. For any invariant polynomial $\phi$, the characteristic form $\phi\left(D_{F}\right)-\phi\left(D_{E}\right)$ extends as a closed, smooth differential form on $X$. Suppose the singularities $\Sigma_{i}$ of the bundle map $\alpha: E \mapsto F$ are orientable, closed submanifolds of $X$. Furthermore, assume that $\alpha$ is extendable. The condition that the $\Sigma_{i}$ 's are orientable arises in many natural settings, for instance, orientation preserving finite group actions on a compact manifold. The residues $\operatorname{Res}_{\phi, i}$ are then closed currents on $X$ (see Remark 5.4). More precisely, by Theorem 5.1, $\operatorname{Res}_{\phi, i} \in H^{2 \operatorname{deg} \phi-e_{i}}(X ; \mathbb{R})$, where $e_{i}=\operatorname{codimRes}_{\phi, i}$. We then have the following immediate corollary.

Corollary 11.1. Let $\alpha: E \mapsto F$ be an extendable bundle map with singularities $\Sigma_{i}$ that are orientable, closed submanifolds of $X$. Assume that rankE $=\operatorname{rankF}$. Let codim $\Sigma_{i}=e_{i}$. Suppose that the cohomology groups $H^{2 \text { deg } \phi-e_{i}}(X ; \mathbb{R})=0$ for each $i$. Then $\phi\left(D_{F}\right)$ and $\phi\left(D_{E}\right)$ are cohomologous.

Proof. By (5.1) we have that

$$
\phi\left(D_{F}\right)-\phi\left(D_{E}\right)=\sum_{i=1}^{l} \operatorname{Res}_{\phi, i}\left[\Sigma_{i}\right]+d T .
$$


Since $H^{2 \operatorname{deg} \phi-e_{i}}(X ; \mathbf{R})=0$, each $\operatorname{Res}_{\phi, i}$ is exact, i.e.,

$$
\operatorname{Res}_{\phi, i}=d S_{i}
$$

for some $S_{i} \in \Omega^{2 \operatorname{deg} \phi-e_{i}-1}(X ; \mathbb{R})$. Hence

$$
\phi\left(D_{F}\right)-\phi\left(D_{E}\right)=d\left(\sum_{i=1}^{l} S_{i}\left[\operatorname{Res}_{\phi, i}\right]+T\right)
$$

which proves the assertion.

This corollary can be viewed as an obstruction theorem to the existence of bundle maps with orientable singularities of a certain codimension.

Corollary 11.2. Let $E$ and $F$ be vector bundles over $X$ where rankE $=$ rank $F$. Suppose that $\phi\left(D_{E}\right)$ and $\phi\left(D_{F}\right)$ are not cohomologous for a given invariant polynomial $\phi$. Also suppose that $H^{2 \operatorname{deg} \phi-k}(X ; \mathbb{R})=0$ for some $k<2$ deg $\phi$. Then there cannot exist a bundle map $\alpha: E \mapsto F$ with an orientable, closed singularity $\Sigma$ of codimension $k$.

Proof. Assume that such an $\alpha$ exists. We can always homotope $\alpha$ such that it becomes extendable with the same singularity $\Sigma$. By Corollary $11.1 \phi\left(D_{E}\right)$ and $\phi\left(D_{F}\right)$ are cohomologous, which contradicts the assumptions.

We also the have the following.

Corollary 11.3. Let $\alpha: E \mapsto F$ be an extendable bundle map with singularities $\Sigma_{i}$, where rankE $=\operatorname{rankF}$ and $\operatorname{codim} \Sigma_{i}=e_{i}$. Let $\phi$ be an invariant polynomial such that $2 \operatorname{deg} \phi<\max \operatorname{codim} \Sigma_{i}$. Then $\phi\left(D_{E}\right)$ and $\phi\left(D_{F}\right)$ are cohomologous.

Proof. Again by Theorem 5.1, if $2 \operatorname{deg} \phi<\max \operatorname{codim} \Sigma_{i}$ then $\operatorname{Res}_{\phi, i}=0$ for each $i$. Hence

$$
\phi\left(D_{F}\right)-\phi\left(D_{E}\right)=d T .
$$




\section{Singularities of Maps.}

Let $X$ and $Y$ be smooth Riemannian manifolds of equal dimension and consider a smooth mapping

$$
f: X \rightarrow Y \text {. }
$$

Consider the differential map

$$
d f: T X \rightarrow f^{*} T Y .
$$

Here we endow $T X$ and $f^{*} T Y$ with the standard riemannian connections, normalized at the singularities of $d f$. We are now in the standard setting where $E=T X$ and $F=f^{*} T Y$. Let $p_{k}(Y)$ and $p_{k}(X)$ be the k-th Pontryjagin forms in the normalized riemannian curvatures of $X$ and $Y$.

A straightforward application of the main residue theorem yields the following result.

Theorem 12.1. Suppose that $f: X \rightarrow Y$ is a smooth map between compact oriented riemannian n-manifolds. Suppose that the differential map df has submanifold singularities $\Sigma_{i}$ and is extendable. Then for any $k \leq n / 4$

$$
f^{*} p_{k}(Y)-p_{k}(X)=\sum_{i=1}^{l} \operatorname{Res}_{p_{k}, i}\left[\Sigma_{i}\right]+d T
$$

and $\operatorname{Res}_{\phi, i}=0$ if $\operatorname{codim} \Sigma_{i}>4 k$.

Proof. We just observe that $p_{k}\left(f^{*} T Y\right)=f^{*} p_{k}(T Y)$ and apply Theorem 5.1 .

An interesting special case occurs when $\mathrm{n}=4 \mathrm{k}$. This yields a $4 \mathrm{k}$-dimensional analogue of the classical Riemann-Hurwitz theorem [M1],[M2],[R].

Corollary 12.2. Suppose that $f: X \rightarrow Y$ is a smooth map between compact oriented 4k-manifolds. Suppose that the differential map df has submanifold singularities $\Sigma_{i}$ and is extendable. Then

$$
M_{f} p_{Y}-p_{X}=\sum_{i=1}^{l} \int_{\Sigma_{i}} R e s_{p_{k}, i}
$$

where $M_{f}$ is the degree of the map $f$ and $p_{Y}$ and $p_{X}$ are the top Pontryjagin numbers of the manifolds $X$ and $Y$ respectively. 
More generally we consider $\wp$, a homogeneous polynomial of weight $\mathrm{k}$ in $\mathrm{k}$ indeterminates. The associated Pontryjagin number to $\wp$ of a compact, oriented manifold $X$ of dimension $4 \mathrm{k}$ is defined to be

$$
\wp(X)=\int_{X} \wp\left(p_{1}(X), \ldots, p_{k}(X)\right) .
$$

Then we have the following corollary.

Corollary 12.3. Suppose that $f: X \rightarrow Y$ is a smooth map between compact oriented 4k-manifolds. Suppose that the differential map df has submanifold singularities $\Sigma_{i}$ and is extendable. Then

$$
M_{f} \wp(Y)-\wp(X)=\sum_{i=1}^{l} \int_{\Sigma_{i}} \operatorname{Res}_{\wp, i}
$$

where $M_{f}$ is the degree of the map $f$ and $\wp(X)$ and $\wp(Y)$ are the Pontryjagin numbers associated to $\wp$ of the manifolds $X$ and $Y$ respectively.

For a topological approach to these formulae see [N], [GGV].

In particular, by the Hirzebruch signature formula [MS] which states that the signature of a compact, oriented manifold of dimension $4 \mathrm{k}$ is expressible as a polynomial in the Pontyjagin classes through the L-class, we have the following.

Corollary 12.4. Suppose that $f: X \rightarrow Y$ is a smooth map between compact oriented $4 k$-manifolds. Suppose that the differential map df has submanifold singularities $\Sigma_{i}$ and is extendable. Then

$$
M_{f} \operatorname{sig}(Y)-\operatorname{sig}(X)=\sum_{i=1}^{l} \int_{\Sigma_{i}} \operatorname{Res}_{s i g, i}
$$

where $M_{f}$ is the degree of the map $f$ and $\operatorname{sig}(X)$ and $\operatorname{sig}(Y)$ are the signatures of the manifolds $X$ and $Y$ respectively.

Remark 12.5. The results in this section apply naturally to branched coverings

$$
\pi: X \mapsto Y
$$

branched along a submanifold $\Sigma$ of codimension 2 in $Y$ and also to finite group actions on a compact manifold. In a subsequent paper we study these important cases where the singularities are of nongeneric codimension in more detail, providing explicit calculations of the residues that appear in the formulae above. 
Remark 12.6. It is clear that similar formulae hold for the case of maps between complex manifolds.

\section{CR-Singularities.}

The methods introduced above yield interesting results in CR-geometry. Consider an immersion $f: X \hookrightarrow Z$ of a real manifold $X$ into a complex manifold $Z$ where

$$
\operatorname{dim}(X)=n=\operatorname{dim}(Z) .
$$

Then the differential map $d f: T X \rightarrow f^{*} T Z$ extends to a complex bundle map

$$
d f: T X \otimes \mathbb{C} \rightarrow f^{*} T Z .
$$

Assume that the bundle map $d f$ has submanifold singularities $\Sigma_{i}$. This corresponds to the loci of points where $f_{*} T_{x} X$ contains a complex subspace having 'excess' dimensions, i.e., more complex tangency than expected. Specifically we have (see $[$ HL2;10.3] for a proof)

\section{Lemma 13.1.}

$$
\bigcup \Sigma_{i}=\left\{x \in X: \operatorname{dim}\left(T_{x} \cap J T_{x} X\right)>0\right\}
$$

where $J$ denotes the complex structure of $Z$.

We now suppose that $X$ carries a Riemannian metric and $Z$ carries a hermitian metric and a complex connection, and we normalize these connections at each $\Sigma_{i}$. Let $p_{i}(X)$ and $c_{i}(Z)$ be the $i$-th Pontryjagin and Chern forms of $X$ and $Z$ resp ectively.

Applying Theorem 5.1 yields the following result.

Theorem 13.2. Let $f: X \hookrightarrow Z$ be an immersion of a real m-manifold into a complex m-manifold. Assume that df has submanifold singularities $\Sigma_{i}$ and that it is extendable. Then

$$
f^{*} c_{2 i}(Z)-p_{i}(X)=\sum_{i=1}^{l} \operatorname{Res}_{\phi, i}\left[\Sigma_{i}\right]+d T .
$$


Proof. Apply Theorem 5.1 to the bundle map $d f$ and observe that

$$
(-1)^{i} c_{2 i}(T X \otimes \mathbb{C})=p_{i}(X) .
$$

Consider the case when $Z=\mathbb{C}^{n}$. This gives the following.

Corollary 13.3. Let $f: X^{n} \hookrightarrow \mathbb{C}^{n}$ be an immersion with the property that $d f$ has submanifold singularities $\Sigma_{i}$ and that it is extendable. Then

$$
p_{i}(X)=\sum_{i=1}^{l} \operatorname{Res}_{\phi, i}\left[\Sigma_{i}\right]+d T .
$$

\section{Finite Singularities and a Generalized Hopf Index Formula.}

The finite singularity sets of r-vector fields and r-plane fields were studied extensively by E.Thomas [T1],[T2], Atiyah [A], and Atiyah-Dupont [AD]. In certain cases they managed to relate these singularities to algebraic invariants of the manifold. We continue the study of finite singularities in the general setting of bundle maps over a compact manifold.

In $\S 9$ we discussed the universal transgression form $\tilde{T}$. We now use the universal construction to prove the following theorem.

Theorem 14.1. Let $\alpha: E \rightarrow F$ be a bundle map over a compact manifold $X$ with isolated finite singularities $\left\{x_{i}\right\}$. Assume that $\alpha$ is normalized at each $x_{i}$. Let $\operatorname{rank}(E)=m$ and $\operatorname{rank}(F)=n$. Then for any invariant polynomial $\phi$,

$$
\int_{X} \phi\left(D_{F}\right)-\phi\left(D_{E} \oplus D_{I^{\perp}}\right)=\sum_{i=1}^{l} \int_{S^{i}} \alpha^{*} \tilde{T}
$$

where $\tilde{T} \in \Omega^{\text {odd }}\left(V_{n, m}\right)$. Here $V_{n, m}$ is the Stiefel manifold of $m$-frames in $\mathbb{R}^{n}$ and the $S^{i}$ are small spheres around the points $\left\{x_{i}\right\}$.

Proof. We recall that $T=\alpha^{*} \tilde{T}$ outside $x_{i}$. Since $E$ and $F$ are normalized at $x_{i}$ then near $x_{i}$

$$
\operatorname{Hom}^{\times}(E, F)=H o m^{*}\left(\mathbb{R}^{m}, \mathbb{R}^{n}\right)=V_{n, m} .
$$


Now we apply Theorem 5.1 and integrate over the manifold. Since $\alpha$ is normalized at each $x_{i}$, by Lemma 7.1 we do not need to take a limit for the residue.

This theorem is a bundle map analogue of the classical Hopf index formula. It has the following interesting corollary.

Corollary 14.2. Consider a map $\alpha: E \rightarrow F$ over a compact manifold $X$ of dimension $4 n$ with isolated finite singularities, where $\operatorname{rank}(E)=2$ and $\operatorname{rank}(F)=n$. Suppose that $\alpha$ is normalized at the singularities. Then

$$
\int_{X} p_{n}(F)-p_{1}(E) p_{n-1}\left(I^{\perp}\right)=0 .
$$

In particular, if $p_{1}(E)=0$ then $p_{n}(F)=0$. Here $p_{i}$ denotes the $i$-th Pontryjagin class.

Proof. This is just a consequence of dimension. By the theorem above $\tilde{T}$ is a differential form of degree $4 \mathrm{n}-1$ on $V_{4 n, 2}$. But $\operatorname{dim} V_{4 n, 2}=4 n-3$ and hence $\tilde{T}=0$.

Remark 14.3. The result above is not a consequence of obstruction theory because in general, $\pi_{4 n-1}\left(V_{4 n, 2}\right) \neq 0$.

We would like to know when $\tilde{T}$ is closed near $x_{i}$. Then the residue can be interpreted using cohomology of $V_{n, m}$. For this we use the following construction.

Let $G_{n, m}$ be the Grassmann manifold of m-planes in $\mathbb{R}^{n}$ and let $\rho$ : $V_{n, m} \rightarrow G_{n, m}$ be the standard fiber map. Also let $\tau$ be the tautological bundle over $G_{n, m}$ and $\tau^{\perp}$ be its dual. Give $\tau^{\perp}$ the connection induced by projection. This construction yields the following lemma.

Lemma 14.4. $\tilde{T}$ is closed near $x_{i}$ iff $\phi\left(\tau^{\perp}\right)=0$ on $G_{n, m}$.

Proof. We can write

$$
\phi\left(\mathbb{R}^{n}\right)-\phi\left(\mathbb{R}^{m} \oplus \tau^{\perp}\right)=d \hat{T} \quad \text { on } \quad G_{n, m} .
$$

By construction $\tilde{T}=\rho^{*} \hat{T}$. The equation above reduces to

$$
\phi\left(\tau^{\perp}\right)=d \hat{T} .
$$


Therefore, if $\phi\left(\tau^{\perp}\right)=0$ on $G_{n, m}$, then $\hat{T}$ is closed and hence $\tilde{T}$ is also closed.

The lemma above yields the following corollary.

Corollary 14.5. Let $\alpha: E \rightarrow F$ be a bundle map over a compact manifold $X$ with isolated finite singularities $\left\{x_{i}\right\}$. Assume that $\alpha$ is normalized at each $x_{i}$. Let $\operatorname{rank}(E)=m$ and $\operatorname{rank}(F)=n$.

(1) If $2 i>n-m$ and $\phi \equiv p_{i}$, then $\tilde{T} \in H^{4 i-1}\left(V_{n, m}\right)$.

(2) If $\phi$ is a multiplicative polynomial and $\phi_{i}$ is its $i$-th degree term such that $2 i>m(n-m)$, then $\tilde{T} \in H^{2 i-1}\left(V_{n, m}\right)$.

Proof. For (1) we observe that $\operatorname{rank}\left(\tau^{\perp}\right)=n-m$. If $2 i>n-m$ then

$$
0=p_{i}\left(\tau^{\perp}\right)=d \hat{T} .
$$

Hence $\hat{T}$ is closed and $\tilde{T} \in H^{4 i-1}\left(V_{n, m}\right)$.

(2) is again a consequence of dimension. Note that $\operatorname{dim} G_{n, m}=m(n-m)$. If $2 i>m(n-m)$ then

$$
0=\phi\left(\tau^{\perp}\right)=d \hat{T},
$$

because it is a differential form of degree higher than the dimension of $G_{n, m}$. Therefore $\hat{T}$ is closed and hence $\tilde{T} \in H^{2 i-1}\left(V_{n, m}\right)$.

\section{Clifford and Spin Bundles.}

Let $\pi: F \mapsto X$ be a 2 n-dimensional vector bundle with spin structure (for a complete discussion of the following constructions see [LM]). Assume that $F$ is provided with a connection $D$. Let $\mathcal{S}$ denote the complex spinor bundle associated to $F$ and let $D_{\mathcal{S}}$ be the connection on $\mathcal{S}$ induced from the one on $F$. There is a canonical decomposition

$$
\mathcal{S}=\mathcal{S}^{+} \oplus \mathcal{S}^{-}
$$

by the complex volume form. Suppose that we are given an odd form $\alpha$, i.e., $\alpha \in \Gamma\left(\bigwedge^{\text {odd }}(F)\right)$. Then $\alpha$ is a bundle map from $\mathcal{S}^{+}$to $\mathcal{S}^{-}$. Assume that

$$
\alpha: \mathcal{S}^{+} \mapsto \mathcal{S}^{-}
$$


has closed submanifold singularities $\Sigma_{i}$. Then again we are in the setting of Theorem 5.1, with rank $\mathcal{S}^{+}=\operatorname{rank} \mathcal{S}^{-}$. Furthermore, if $E$ is any complex vector bundle with complex connection $D_{E}$, we let

$$
D_{\mathcal{S} \otimes E}=D_{\mathcal{S}} \otimes 1+1 \otimes D_{E}
$$

denote the tensor product connection on $\mathcal{S} \otimes E$ and normalize this connection at $\Sigma_{i}$. This connection observes the splitting of the spinor bundle. The odd form $\alpha$ is again a bundle map from $\mathcal{S}^{+} \otimes E$ to $\mathcal{S}^{-} \otimes E$ and we have the following.

Corollary 15.1. Let $(F, D)$ and $\left(\mathcal{S} \otimes E, D_{\mathcal{S} \otimes E}\right)$ be as above. Suppose that $\alpha \in \Gamma\left(\bigwedge^{\text {odd }}(F)\right)$ has closed submanifold singularities $\Sigma_{i}$ as a bundle map $\alpha: \mathcal{S}^{+} \mapsto \mathcal{S}^{-}$and is extendable. Then

$$
\operatorname{ch}\left(D_{\mathcal{S}^{+} \otimes E}\right)-\operatorname{ch}\left(D_{\mathcal{S}^{-} \otimes E}\right)=\sum_{i=1}^{l} \operatorname{Res}_{c h, i}\left[\Sigma_{i}\right]+d T .
$$

Remark 15.2. The same formula holds when $F$ is $\operatorname{spin}^{c}$.

Even if $F$ is not spin or $\operatorname{spin}^{c}$, we can derive an interesting residue formula by considering the complex Clifford bundle $C \ell$ associated to $F$. Again we have a decomposition

$$
C \ell=C \ell^{+} \oplus C \ell^{-}
$$

and an odd form $\alpha \in \Gamma\left(\bigwedge^{\text {odd }}(F)\right)$ acts as a bundle map

$$
\alpha: C \ell^{+} \otimes E \mapsto C \ell^{-} \otimes E
$$

where $E$ is any complex vector bundle with complex connection $D_{E}$. Again we let

$$
D_{C \ell \otimes E}=D_{C \ell} \otimes 1+1 \otimes D_{E}
$$

denote the tensor product connection on $C \ell \otimes E$ and we normalize this connection at $\Sigma_{i}$, the submanifold singularities of $\alpha$. As before this connection preserves the splitting of $C \ell$. We immediately have

Corollary 15.3. Let $(F, D)$ and $\left(C \ell \otimes E, D_{C \ell \otimes E}\right)$ be as above. Suppose that $\alpha \in \Gamma\left(\bigwedge^{\text {odd }}(F)\right)$ has closed submanifold singularities as a bundle map $\alpha: C \ell^{+} \otimes E \mapsto C \ell^{-} \otimes E$ and is extendable. Then

$$
\operatorname{ch}\left(D_{C \ell^{+} \otimes E}\right)-\operatorname{ch}\left(D_{C \ell^{-} \otimes E}\right)=\sum_{i=1}^{l} \operatorname{Res}_{c h, i}\left[\Sigma_{i}\right]+d T .
$$


Remark 15.4. An explicit calculation of the residue in these two cases would be useful because it would yield analogues of Grothendieck - Riemann - Roch [AH].

\section{References.}

[A] M.F. Atiyah, Elliptic Operators and Singularities of Vector Fields, Actes Congres Inter. Math., Tome 2, 207-209.

[AD] M.F. Atiyah and S.L. Dupont, Vector Fields with Finite Singularities, Acta Math. 128 (1972), 1-40.

[AH] M.F. Atiyah and F. Hirzebruch, The Riemann-Roch theorem for analytic embeddings, Topology 1 (1962), 151-166.

[BoC] R. Bott and S.S. Chern, Hermitian vector bundles and the equidistribution of the zeros of their holomorphic sections, Acta. Math. 114 (1968), 71-112.

[F] H. Federer, Geometric Measure Theory, Springer-Verlag, 1969.

[GGV] S. Gitler, J.F. Glazebrook and A. Verjovsky, On the Generalized RiemannHurwitz Formula, Bol. Soc. Mat. Mexicana 30,1 (1985), 1-11.

[H] H. Hopf, Vectorfelder in n-dimensionalen Mannigfaltigkeiten, Math Ann. 96 (1927), 225-260.

[HL1] F.R. Harvey and H.B. Lawson, A Theory of Characteristic Currents Associated with a Singular Connection, Asterisque 213 (1993), 1-268.

[HL2] Reese Harvey and H. Blaine Lawson, Geometric Residue Theorems, American J. Math 117 (1995), 829-873.

[HS] F.R. Harvey and S. Semmes, Zero Divisors of atomic functions, Ann.Math 135 (1992), 567-600.

[LM] H.B. Lawson and M. Michelsohn, Spin Geometry, Princeton University Press, Princeton, New Jersey (1989).

[M1] R. MacPherson, Singularities of Vector Bundle Maps, Proceedings of Liverpool Singularities Symposium, I, Lect. Notes in Math., vol. 192, SpringerVerlag, 316-318.

[M2] R. MacPherson, Generic Vector Bundle Maps, Dynamical Systems, Proceedings of Symposium-University of Bahia, Salvador 1971, Academic Press (1973), 165-175. 
[MS] J. Milnor and J.D. Stasheff, Characteristic Classes, Princeton University Press, Princeton, New Jersey (1974).

[N] Ngo Van Que, Generalization de la formula de Riemann-Hurwitz, Canad. J. Math. 24 (1972), 761-767.

[R] F. Ronga, Le calcul da la classe de cohomologie duale a $\Sigma$, Proceedings of Liverpool Singularities Symposium, I, Lecture Notes in Math., vol. 192, Springer-Verlag, 1971, 313-315.

[S] P. Shanahan, The Atiyah-Singer Index Theorem, An Introduction, Lect. Notes in Math. 638, Springer-Verlag, New York, 1978.

[T1] E. Thomas, Vector Fields on Manifolds, Bull.Americ.Math.Soc. 75 (1969), 643-683.

[T2] E. Thomas, Fields of Tangent k-Planes on Manifolds, Invent.Math 3 (1967), 334-347.

57 Shelton Street

COVENT GARDEN

LONDON WC2H 9HE

ENGLAND

E-mail: sunil@wspc.demon.co.uk 